\title{
Previsão do campo de vento empregando o modelo WRF para a análise do potencial eólico
}

Wind forecast employing the WRF model for a wind potential analysis

\author{
Mariana Medeiros Ghizoni ${ }^{1}$, Silvana Maldaner ${ }^{2}$, Greice Scherer Ritter ${ }^{3}$ e Alcimoni Nelci Comin ${ }^{4}$ \\ 1,2,3,4,Universidade Federal de Santa Maria, Cachoeira do Sul, Brasil \\ mari.ghizoni@hotmail.com; silvana.maldaner@ufsm.br
}

\section{Resumo}

O WRF (Weather Research and Forecast) é um software de modelagem numérica aplicado à atmosfera. Este modelo foi desenvolvido para a previsão do tempo e investigação de fenômenos atmosféricos de mesoescala. O WRF é de domínio público e de distribuição gratuita, possuindo diferentes aplicações que vão do campo da meteorologia até a engenharia, podendo ser aplicado tanto em situações de atmosfera idealizada quanto de atmosfera real. Atualmente, o campo de vento simulado por este software vem sendo utilizado como dado real. Assim, o modelo WRF passou a ser empregado em análises de vento para geração de energia é́lica. Este trabalho, tem como objetivo simular o campo de vento empregando o modelo WRF para a cidade de Cachoeira do Sul, Rio grande do Sul, Brasil.

Palavras-chave: Modelo WRF; Vento; Energia Eólica

\section{Abstract}

The WRF (Weather Research and Forecast) is a numerical modeling applied to the atmosphere. This model was developed for weather forecasting and investigation of atmospheric mesoscale phenomena. The WRF is a public domain and free distribution, with different applications ranging from the field of meteorology to engineering, and can be applied in situations of idealized atmosphere or real atmosphere. Presently, the wind field simulated by this model has been used as real data. Thus, the WRF model started to be employed in wind analysis for wind power generation. In this work, the wind field was simulated using the WRF model, in Cachoeira do Sul, Rio Grande do Sul, Brazil.

Keywords: WRF; Wind field; Wind energy; 


\section{Introdução}

No Brasil a previsão do vento em período de curto prazo é realizada pelo uso de modelos atmosféricos de mesoescala (RAMOS; LYRA; DA SILVA RAMOS, 2013). Um modelo bem conhecido para prever o campo de vento é o WRF (Weather Research and Forecast). O WRF é um modelo não hidrostático de previsão do tempo e clima, de alto desempenho computacional, prevendo condições atmosféricas futuras com dias de antecedência. A física do modelo é baseada nas equações de conservação de massa, explicitando as variações de energia, momentum e umidade do ar (RAMOS; LYRA; DA SILVA RAMOS, 2013).

Segundo Carvalho (2009), o modelo WRF pode ser empregado para simulações do módulo da velocidade do vento com intuito de determinar o regime local de ventos, e desta forma, o recurso eólico disponível. Nesta época, observou-se que o modelo subestimava as magnitudes do vento em até $30 \%$.

Recentemente, o WRF passou a ser empregado em simulações para previsão de vento com fins de geração de energia eólica, passando a ser utilizado para auxiliar na identificação de locais com alto potencial eólico.

Uma previsão de vento com WRF para o estado de Alagoas foi realizada por Ramos, Lyra, Da Silva Ramos (2013), que verificou que o modelo possui alta precisão nas simulações dos valores médios de velocidade e direção do vento.

Baseados no argumento de que raramente se têm dados de medições do vento, Tuchtenhagen, Basso e Yamasaki (2014) desenvolveram uma metodologia para avaliar o potencial eólico para o Brasil no período de um ano. Na conclusão geral da análise realizada, os autores apontam como áreas mais favoráveis as regiões próximas do litoral do nordeste e sul do país.

Na previsão da magnitude da velocidade do vento, Oliveira (2017) avaliou a performance do modelo WRF na região do litoral de Santa Catarina. Os resultados mostraram que o WRF prevê de forma adequada as tendências de aumento/diminuição da velocidade do vento.

Simulações das condições de vento no estado da Paraíba, através do modelo WRF, foram realizadas por Oliveira e Souza (2017). Estes observaram uma boa concordância entre os perfis de vento simulados pelo modelo WRF e os dados de vento coletados nas torres anemométricas.

O WRF foi usado para estimar a velocidade do vento na região central do estado de Alagoas, por Santos da Silva et al. (2018). O modelo estimou com precisão as médias mensais e diárias da velocidade do vento, e verificou-se que as incertezas geradas pelo modelo aumentam para estimativa de médias de períodos mais curtos, como médias horárias.

Como destacado pelos autores, o modelo WRF é uma ferramenta computacional para previsão do campo de vento, tanto em superfície, quanto em níveis superiores. Assim, neste estudo emprega-se o modelo WRF para simular o campo de vento na cidade de Cachoeira do Sul, RS, Brasil.

\section{Metodologia e resultados}

Neste trabalho, simulou-se através do modelo WRF, o campo de vento para o dia 20 de maio de 2017, para a cidade de Cachoeira do Sul, no estado do Rio Grande do Sul. O domínio da simulação é descrito na Figura 1. A simulação foi realizada com uma resolução de 12 x $12 \mathrm{~km}$ e 4 x 4 km, conforme Figura 1:

Na configuração do WRF, foram selecionados os seguintes critérios: Esquema microfísico de WSM6; parametrização de cumulus Kain (2004) na grade externa (na grade interna não foi utilizada parametrização); esquema de rápida transferência radiativa (RRTM) para a radiação de onda longa, com baseem Mlaweret al. (1997); o esquema de Dudhia (1989) para a radiação de onda curta; o esquema de Quasi-Normal Scale Elimination (QNSE) proposto por Sukoriansky et al. (2005) para a 
camada limite planetária; e o esquema Noah para a superfície terrestre (Niuet al. 2011). As condições iniciais e de contorno foram obtidas do NCEP- FNL com $1^{\circ}$ de resolução espacial. As simulações foram feitas com 30 níveis na vertical.

Figura 1 - Domínio no modelo WRF

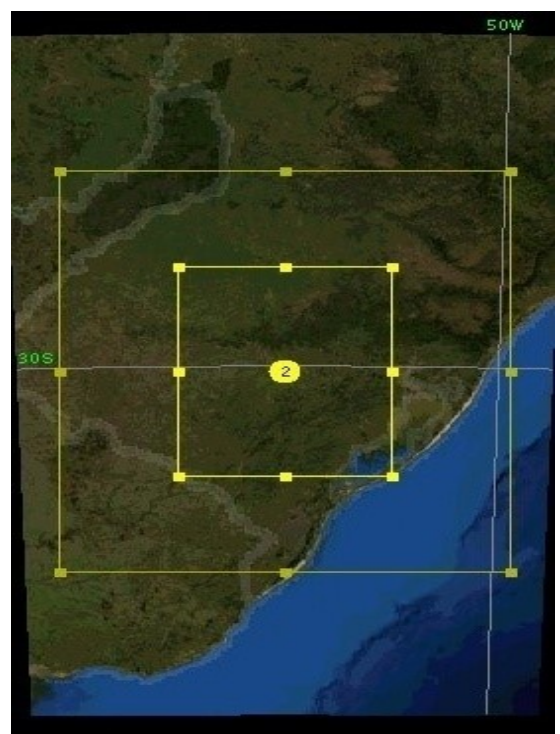

A Figura 2 mostra os resultados da simulação de velocidades de vento zonal (figura 2a) e vento meridional (figura 2b), em função da altitude, para Cachoeira do Sul, em linha preta para as 9 horas da manhã, e em linha azul para as 15 horas do dia 17 de Maio de 2017.
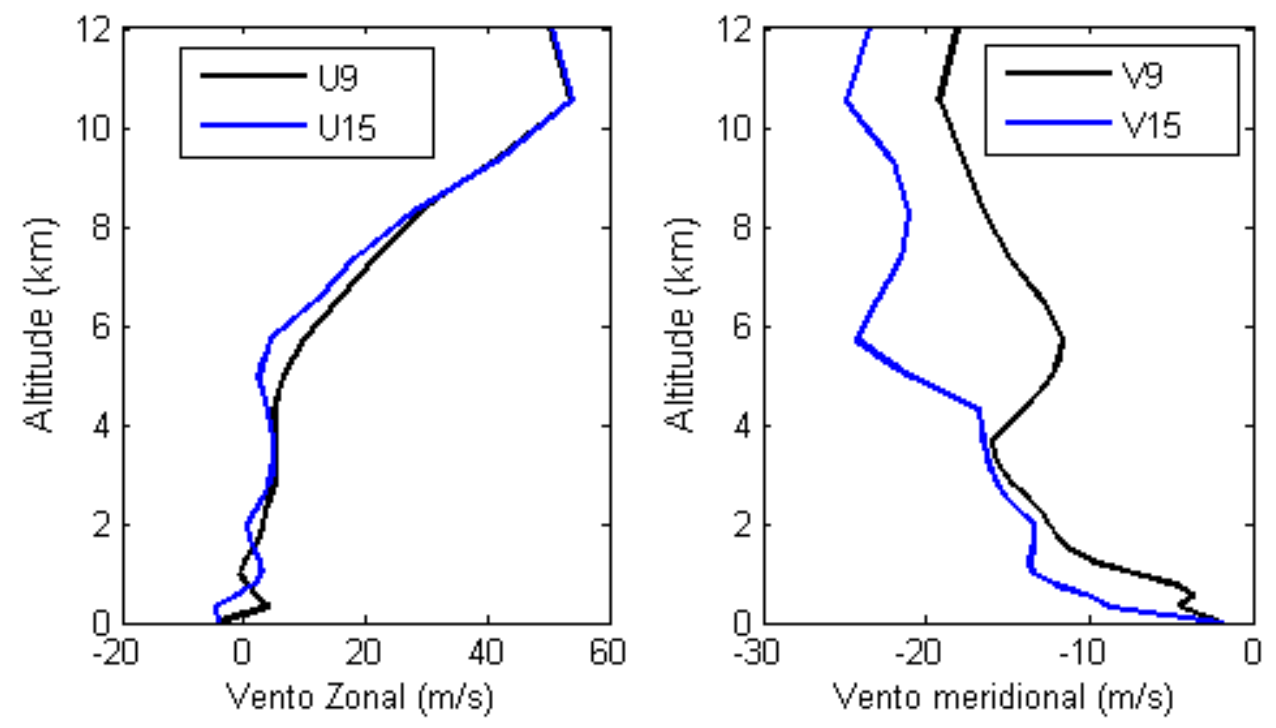
A Figura 2 mostra, da esquerda para direita, o vento na direção x e y em função da altitude em Km. Pode-se observar que próximo à superfície, as magnitudes do vento são abaixo de $5 \mathrm{~m} / \mathrm{s}$, tanto para as 9 horas da manhã quanto para as 15 horas. A Figura 3 mostra a variação da temperatura com a altitude e pode-se observar que não ocorreram variações significativas das 9 (linha preta) para as 15 horas (linha azul).

Figura 3 - Temperatura simulada pelo modelo WRF.

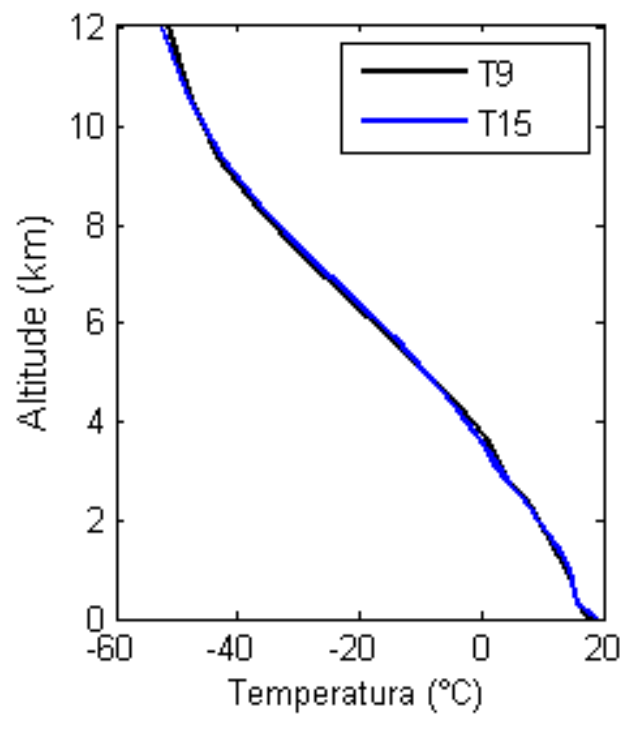

A figura 4 apresenta a variação da umidade relativa com a altitude. Pode-se observar que os valores de umidade relativa, em superfície, são bastante elevados, característica da cidade neste período do ano.

Figura 4 - Umidade relativa simulada pelo modelo WRF.

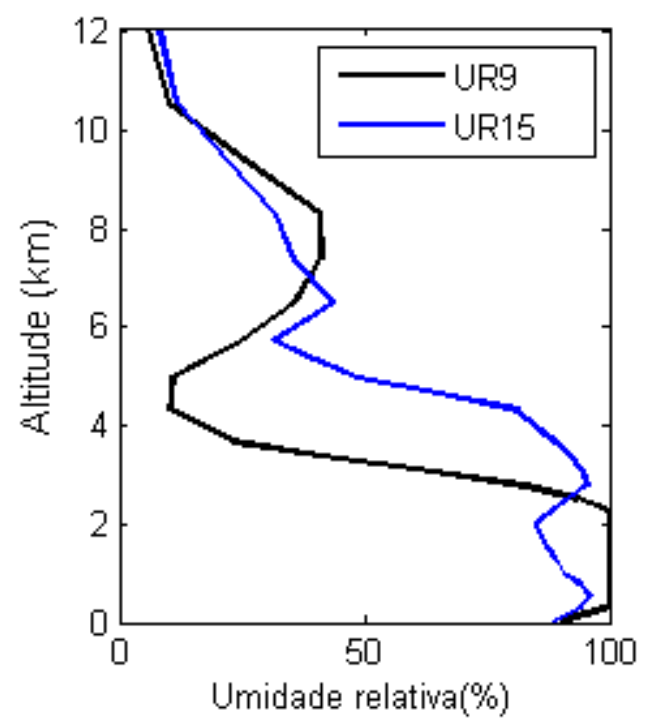


Como a potência do vento é definida a partir da sua velocidade elevada ao cubo (SANSIGOLO, 2005), conhecendo-se o campo de vento de uma região pode-se estimar o potencial eólico da mesma.

\section{Conclusões}

Neste trabalho, simulou-se o campo de vento para a cidade de Cachoeira do Sul para dia 20 de maio de 2017. Como resultado, obteve-se baixas magnitudes da velocidade do vento em superfície, indicando baixa potência do vento. Simulações WRF mais detalhadas (simulações com maior resolução) devem ser realizadas para a região em questão, para uma conclusão definitiva sobre o comportamento do vento com a altura.

\section{Agradecimentos}

Os autores agradecem à Fundação de Amparo à Pesquisa do Estado do Rio Grande do Sul -FAPERGS (edital 01/2017 - ARD), aoConselho de Desenvolvimento Científico e Tecnológico - CNPq, à Coordenadora do Polo UAB- Cachoeira do Sul - Rosane Aparecida Brendler Keller, à Prefeitura Municipal de Cachoeira do Sul e ao Programa de Auxílio à pesquisa de recém-doutores "FIPE -Júnior/UFSM".

\section{Referências}

CARVALHO, DJS. Optimização do modelo numérico de previsão do tempo WRF no contexto de previsão e produção de energia eólica, em Aveiro/Portugal[dissertation]. Aveiro: Universidade de Aveiro; 2009. 53p.

DA SILVA RAMOS DN, LYRA DF,DA SILVA JÚNIOR RF, SALUSTIANO R. Previsão do vento utilizando o modelo atmosférico WRF para o estado de Alagoas. Revista Brasileira de Meteorologia. 2013; 28(2): 163-172.

DA SILVA SMS, LYRA RFF, Junior RSS, da Cruz NSD, Silva SS. Avaliação do Desempenho do Modelo WRF para Prognóstico do vento na Região Central de Alagoas - Craíbas.Ciência e Natura. 2018; 40:187-192.

DUDHIA J. NumericalstudyofconvectionobservedduringtheWinterMonsoonExperimentusing a mesoscaletwo dimensionalmodel. JournaloftheAtmosphericSciences. 1989;46:3077-3107.

KAIN, J.S.TheKain - Fritschconvectiveparameterization: AnUpdate. J. Appl. Meteorol. 2004; 43(1):170-181.

MLAWER, EJ. et al. Radiativetransfer for inhomogeneousatmospheres: RRTM, a validatedcorrelated-kmodel for thelongwave. JournalofGeophysicalResearch: Atmospheres. 1997; 102: 16663-16682.

NIU GY, YANG ZL, MITCHELL KE, CHEN F,EK MB, BARLAGE M et al. ThecommunityNoahandsurfassemodelwithmultiparameterizationoptions (Noah-MP): 1.Modeldescriptionandev aluationwithlocal-scalemeasurements. JournalofGeophysicalResearch. 2011;116:1-19.

OLIVERA, AFS. Avaliação do Modelo WRF na Previsibilidade do Vento para Geração de Energia Eólica na Região de Laguna-SC [monography]. Florianópolis: Departamento de Física/UFSC; 2017.

Oliveira SS, SOUZA EP. Análise de Modelos de Mesoescala para Caracterização do Potencial Eólico do Estado da Paraíba. Revista Brasileira de Meteorologia. 2017; 32(2):77-291.

SANSIGOLO, CA. Distribuições de probabilidade de velocidade e potência do vento. Revista Brasileira de Meteorologia. 2005; 20(2): 207-214. 
SUKORIANSKY, S.; GALPERIN, B.; STAROSELSKY, I. A quasi normal scaleeliminationmodelofturbulentflowswithstablestratification. Phys. Fluids, 2005:17, 1-28.

TUCHTENHAGEN P,BASSO J, YAMASAKI Y. Avaliação do potencial eólico no Brasil em 2011. Ciência e Natura. 2014; 36(2): 390-401. 